American Journal of Applied Sciences 4 (11): 912-917, 2007

ISSN 1546-9239

(C) 2007 Science Publications

\title{
Open Circuit Field Distribution and Induced Voltage of a Cylindrical Permanent Magnet Linear Generator
}

\author{
Wijono and Hamzah Arof \\ Department of Electrical Engineering, Faculty of Engineering, University of Malaya \\ 50603 Kuala Lumpur, Malaysia
}

\begin{abstract}
This paper deals with the analytical computation of the magnetic field of a cylindrical permanent magnet linear generator. It offers an alternative solution to the magnetic field problem. A new approach to solve Poisson equation using Struve function is introduced and it offers a simple but accurate analytical estimation to the calculation of the open circuit flux density and induced voltage. The finite element analysis simulation is performed to validate the analytical calculation.
\end{abstract}

Key Words: linear generator, permanent magnet, analytical method, Struve function, Halbach, electromagnetic field, finite element analysis

\section{INTRODUCTION}

A linear generator is a machine that converts linear mechanical energy into electrical energy. The machine is directly driven by a linear prime mover. It is different from its rotary counterpart in a way that the design of a linear machine mostly follows the characteristic of the prime mover.

The linear generator provides electrical power as a stand alone or as an alternator of a power generation system. A wide range of output power can be had from linear generators from a few watts for a flashlight to a few megawatts from a tidal wave power for normal utilities.

Unlike rotary machines, limited publications on the linear generator show that research on linear generators has not reached the saturation level and therefore there is still a lot of space for research and development. This paper presents a calculation of the flux distribution of a linear generator. Maxwell and Poisson equations are used to compute magnetic field quantities in all parts of the machine.

A comprehensive analysis of the field distribution of linear machines is presented in ${ }^{[7]}$. Treatment of linear machines equipped with Halbach and quasi or discrete Halbach is outlined as a special case in ${ }^{[5]}$ in detail. Several types of winding construction are also offered. The analysis can also be adapted from the rotary machine studies presented in ${ }^{[14-16]}$.

Wang et. al. ${ }^{[5,7]}$ solve the field equations by integrating of the permanent magnet geometry. The exponential form can also be used to solve the Maxwell and Poisson equation in both rotary ${ }^{[12-16]}$ and linear machines ${ }^{[2]}$. In this paper a direct solution involving a Struve function is proposed. The direct solution takes less time since there is no integral form in the matrix of simultaneous equations, and the whole field source is taken into calculation instead of its surface only. The only requirement needed for the direct solution is just some experience in handling differential equations.

The validity of the direct analytic approach presented in this paper is verified by comparing its solutions to those of finite element method. The calculation of the unknown parameters is made easy by taking advantage of the symmetry of the cylindrical machine. A 2D axisymmetry model is set up to give a simpler machine representation of the real 3D object.

\section{MACHINE CONSTRUCTION}

The machine is a long-translator type and constructed of a stator and a translator as shown in the Fig.1. Six windings are located in the stator core. Seven pieces of permanent magnets are mounted on a nonpermeable translator shaft to produce the magnetic field. Those permanent magnets are arranged in a discrete Halbach series, i.e. radially magnetized permanent magnets (RMPMs) and axially magnetized permanent magnets (AMPMs) are stacked alternately.

The machine is run in a reciprocating sinusoidal motion. The system produces a linear three phase ac output. At every translator motion, all windings are always active since the translator is longer than the stator. The machine prototype and its construction is shown in Fig.1.

Corresponding Author: Wijono, Department of Electrical Engineering, Faculty of Engineering, University of Malaya, 50603 Kuala Lumpur, Malaysia 
Am. J. Applied Sci., 4 (11): 912-917, 2007

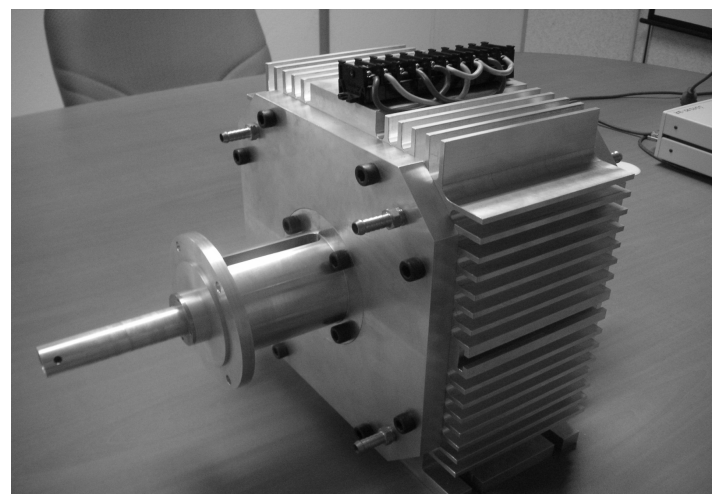

a. Generator Prototype

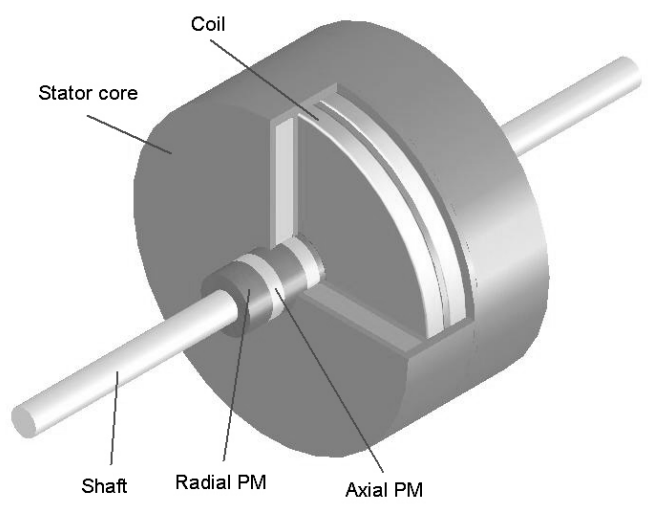

b. Generator Construction

Fig. 1: Linear generator

\section{MODELING AND ANALYSIS}

Fig. 2 shows the cross section of the upper half of the linear generator. As can be seen, the machine can be divided into three regions where region 1 includes the air gap and the winding; region 2 includes the permanent magnet set and region 3 includes the shaft. The region number is noted as a subscript in all equations. The machine specification is listed in Table 1.

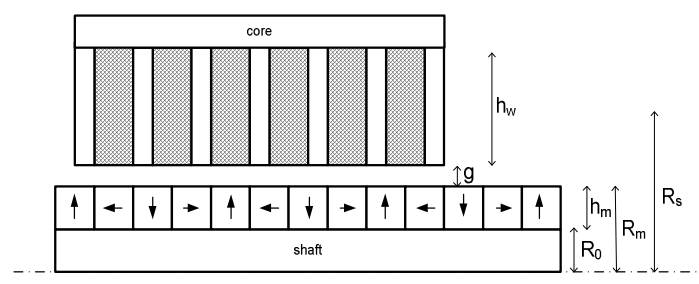

Fig. 2: Cross section of the upper half of the linear generator.

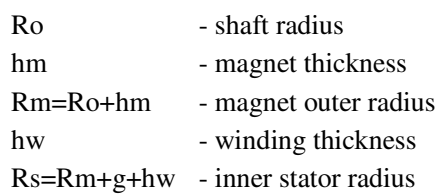

Table 1: Generator specification

\begin{tabular}{lll}
\hline Speed & 3000 & $\mathrm{rpm}$ \\
Stroke & 69 & $\mathrm{~mm}$ \\
$\mathrm{R}_{0}$ & 12.5 & $\mathrm{~mm}$ \\
$\mathrm{~h}_{\mathrm{m}}$ & 12 & $\mathrm{~mm}$ \\
$\mathrm{~h}_{\mathrm{w}}$ & 102 & $\mathrm{~mm}$ \\
AMPM length & 12 & $\mathrm{~mm}$ \\
RMPM length & 22.5 & $\mathrm{~mm}$ \\
$\mathrm{~B}_{\mathrm{r}}$ & 1.12 & $\mathrm{~T}$ \\
$\mu_{\mathrm{r}}$, shaft & 1 & \\
$\mu_{\mathrm{r}}$, core & infinity & \\
\hline
\end{tabular}

In the first step of the analysis, the governing equations are developed while ignoring the existence of stator teeth. The machine is assumed to be infinitively long and has a periodic construction. The permeability of the core is assumed to be infinity while the permeability of the winding, air gap and permanent magnet as well as the shaft is assumed to be one. Next, the effect of teeth is represented by the Carter coefficient. Some other assumptions are made to simplify the calculation.

Flux Distribution: The flux distribution is calculated at any point in all zones. The flux density in the air gap, the winding and the shaft is given by,

$$
\mathbf{B}=\mu_{0} \mathbf{H}
$$

where $\mu_{0}$ is permeability of free space and $\mathbf{H}$ is the field intensity. The flux density in the permanent magnet is given by the combination of the remanent magnetization of permanent magnet and the external field intensity. It can be written as:

$$
\mathbf{B}=\mu_{0} \mu_{r} \mathbf{H}+\mu_{0} \mathbf{M}
$$

where $\mu_{\mathrm{r}}$ is the relative recoil permeability of the magnets and $\mathbf{M}$ is the remanent magnetization vector. It is assumed that the permanent magnet has a linear demagnetization characteristic; so that the magnetization vector is directly related to the field density as:

$$
\mathbf{M}=\frac{\mathbf{B}_{\text {rem }}}{\mu_{0}}
$$


The flux density B can be expressed as the curl of the magnetic vector potential A

$$
\mathbf{B}=\nabla \times \mathbf{A}
$$

Maxwell's and Poisson's equations for the air gap/winding, permanent magnets and shaft are respectively given as,

$$
\begin{aligned}
& \nabla^{2} \mathbf{A}_{1}=0 \\
& \nabla^{2} \mathbf{A}_{2}=-\mu_{0} \nabla \times \mathbf{M} \\
& \nabla^{2} \mathbf{A}_{3}=0
\end{aligned}
$$

The vector potential $\mathbf{A}$ has only one nonzero component, $A_{\theta}$, which is independent of $\theta$ in the cylindrical system. Therefore, Equations (5) until (7) can be written as,

$\frac{\partial}{\partial z}\left(\frac{1}{r} \frac{\partial}{\partial z}\left(r A_{1 \theta}\right)\right)+\frac{\partial}{\partial r}\left(\frac{1}{r} \frac{\partial}{\partial r}\left(r A_{1 \theta}\right)\right)=0$

$\frac{\partial}{\partial z}\left(\frac{1}{r} \frac{\partial}{\partial z}\left(r A_{2} \theta\right)\right)+\frac{\partial}{\partial r}\left(\frac{1}{r} \frac{\partial}{\partial r}\left(r A_{2 \theta}\right)\right)=-\mu_{0} \nabla \times \mathbf{M}$

$$
\frac{\partial}{\partial z}\left(\frac{1}{r} \frac{\partial}{\partial z}\left(r A_{3 \theta}\right)\right)+\frac{\partial}{\partial r}\left(\frac{1}{r} \frac{\partial}{\partial r}\left(r A_{3 \theta}\right)\right)=0
$$

The remanent magnetization vector $\mathbf{M}$ can be written in the form of its components:

$$
\mathbf{M}=M_{r} \mathbf{e}_{r}+M_{z} \mathbf{e}_{z}
$$

where $M_{r} \mathbf{e}_{r}$ represents the contribution of RMPM and $M_{z} \mathbf{e}_{z}$ represents the contribution of AMPM. When the machine uses only one type of those permanent magnets, the remanent magnetization vector will only have one component.

From Equation (4), the flux density B obtained from $\mathbf{A}$ is given by

$$
\begin{aligned}
& B_{z}=\frac{1}{r} \frac{\partial}{\partial r}\left(r A_{\theta}\right) \\
& B_{r}=-\frac{\partial A_{\theta}}{\partial z}
\end{aligned}
$$

In the series form, from Equations (3) and (11) the components of the magnetization vector are decomposed into harmonics,

$$
\begin{aligned}
& M_{r}=\sum_{\eta=1,2, \ldots}^{\infty} M_{r} \eta^{\sin \left(m_{\eta} z\right)} \\
& M_{z}=\sum_{\eta=1,2, \ldots}^{\infty} M_{z} \eta \cos \left(m_{\eta} z\right)
\end{aligned}
$$

where

$$
\begin{aligned}
& m_{\eta}=\frac{(2 \eta-1) \pi}{\tau_{p}} \\
& M_{r \eta}=\frac{4 B_{r e m}}{\mu_{0} \tau_{p} m_{\eta}} \sin \frac{m_{\eta} \tau_{p}}{2} \sin \frac{m_{\eta} \tau_{m r}}{2} \\
& M_{z \eta}=\frac{4 B_{r e m}}{\mu_{0} \tau_{p} m_{\eta}} \sin \frac{m_{\eta} \tau_{m z}}{2}
\end{aligned}
$$

where $\tau_{\mathrm{p}}$ is the permanent magnet pole pitch, $\tau_{\mathrm{mr}}$ and $\tau_{\mathrm{mz}}$ are the length of RMPM and AMPM, respectively. Using those equations, Equations (8) until (10) can be rewritten as

$$
\begin{gathered}
\frac{\partial}{\partial z}\left(\frac{1}{r} \frac{\partial}{\partial z}\left(r A_{1} \theta\right)\right)+\frac{\partial}{\partial r}\left(\frac{1}{r} \frac{\partial}{\partial r}\left(r A_{1} \theta\right)\right)=0 \\
\frac{\partial}{\partial z}\left(\frac{1}{r} \frac{\partial}{\partial z}\left(r A_{2} \theta\right)\right)+\frac{\partial}{\partial r}\left(\frac{1}{r} \frac{\partial}{\partial r}\left(r A_{2} \theta\right)\right)=
\end{gathered}
$$

$\sum_{\eta=1,2, \ldots}^{\infty} P_{\eta} \cos m_{\eta} z$

$$
\frac{\partial}{\partial z}\left(\frac{1}{r} \frac{\partial}{\partial z}\left(r A_{3 \theta}\right)\right)+\frac{\partial}{\partial r}\left(\frac{1}{r} \frac{\partial}{\partial r}\left(r A_{3 \theta}\right)\right)=0
$$

where

$P \eta=-\frac{4 B_{r e m}}{\tau_{p}} \sin m_{\eta} \frac{\tau_{p}}{2} \sin m_{\eta} \frac{\tau_{m r}}{2}$

The boundary conditions of the system exist at the interfaces of different zones. A special boundary is located on the axis of the shaft where vector potential is zero. Six boundary conditions are needed to solve the simultaneous field equations. 


$$
\begin{aligned}
& B_{z 1} \mid r=R_{S}=0 ; \\
& \left.B_{r 1}\right|_{r=R_{m}}=B_{r 2} \mid r=R_{m} ; \\
& B_{r 2}\left|r=R_{0}=B_{r 3}\right| r=R_{0} ; \\
& \left.A_{\theta 3}\right|_{r=0}=0 ; \\
& \left.H_{z 1}\right|_{r=R_{m}}=H_{z 2} \mid r=R_{m} ; \\
& H_{z 2}\left|r=R_{0}=H_{z 3}\right| r=R_{0}
\end{aligned}
$$

Solving Equations (15) through (18) using Equation (19) and rearranging them in terms of vector potential yield

$$
\begin{aligned}
& A_{\theta 1}=\sum_{\eta=1}^{\infty}\left(\begin{array}{c}
A_{\eta 1} \operatorname{BesselI}_{1}\left(m_{\eta} r\right)+ \\
B_{\eta 1} \operatorname{BesselK}_{1}\left(m_{\eta} r\right)
\end{array}\right) \cos \left(m_{\eta} z\right) \\
& A_{\theta 2}=\sum_{\eta=1}^{\infty}\left(\left(\begin{array}{c}
A_{\eta 2} \operatorname{BesselI}_{1}\left(m_{\eta} r\right)+ \\
B_{\eta 2} \operatorname{BesselK}_{1}\left(m_{\eta^{r}}\right)
\end{array}\right) \cos \left(m_{\eta} z\right)+S(r, z)\right) \\
& A_{\theta 3}=\sum_{\eta=1}^{\infty}\left(\begin{array}{l}
A_{\eta 3} \operatorname{BesselI}_{1}\left(m_{\left.\eta^{r}\right)+}\right. \\
B_{\eta 3} \operatorname{BesselK}_{1}\left(m_{\eta^{r}}\right)
\end{array}\right) \cos \left(m_{\eta} z\right)
\end{aligned}
$$

where BesselI $I_{1}$ is modified Bessel functions of the first kind of order 1 and BesselK $\mathrm{K}_{1}$ is modified Bessel functions of the second kind of order 1 .

The term $S(r, z)$ can be any function of $r$ and $z$. In this case, the Struve function is introduced to solve the equation. Solving Equation (21) for $S(r, z)$ yields

$$
S(r, z)=\frac{1}{2} \frac{\pi \cdot \operatorname{StruveL}_{1}\left(m_{\eta} \cdot r\right) \cdot P_{\eta} \cos \left(m_{\eta} z\right)}{m_{\eta}{ }^{2}}
$$

where StruveL $L_{1}$ is the modified Struve function of order 1 .

It is not necessary to include the physical condition of the field source to find the solution. Instead, the functions can be solved directly using common differential equations. Therefore, the matrix of the simultaneous equations will contain simple function instead of integral function.

The flux distributions in all zones are derived from Equations (20) until (22) using the relationship in Equation (4). It yields:

$$
B_{r 1}=\sum_{\eta=1,2, \ldots}^{\infty} m_{n}\left(\begin{array}{l}
A_{\eta 1} \operatorname{BesselI}_{1}\left(m_{\eta} r\right)+ \\
B_{\eta 1} \operatorname{BesselK}_{1}\left(m_{\eta} r\right)
\end{array}\right) \sin \left(m_{\eta} z\right)
$$

$$
\begin{aligned}
& B_{z 1}=\sum_{\eta=1,2, \ldots}^{\infty} m_{\eta}\left(\begin{array}{l}
A_{\eta 1} \operatorname{BesselI}_{0}\left(m_{\eta} r\right)- \\
B_{\eta 1} \operatorname{BesselK}_{0}\left(m_{\eta} r\right)
\end{array}\right) \cos \left(m_{n} z\right) \\
& B_{r 2}=\sum_{\eta=1,2, \ldots}^{\infty}\left(\begin{array}{l}
\frac{1}{2} \frac{\pi \operatorname{StruveL}_{1}\left(m_{\eta} r\right) \cdot P_{\eta} \sin \left(m_{\eta} z\right)}{m_{\eta}}+ \\
m_{n}\left(\begin{array}{l}
A_{\eta 1} \operatorname{BesselI}_{1}\left(m_{\eta^{r}}+\right. \\
B_{\eta 1} \operatorname{BesselK}_{1}\left(m_{\eta^{r}}\right)
\end{array}\right) \sin \left(m_{\eta^{z}}\right)
\end{array}\right)
\end{aligned}
$$

$$
B_{z 2}=\sum_{n=1,2, \ldots}^{\infty}\left(\begin{array}{l}
\frac{1}{2} \frac{\pi \operatorname{StruveL}_{0}\left(m_{\eta} r\right) P_{\eta} \cos \left(m_{\eta} z\right)}{m_{\eta}}+ \\
m_{\eta}\left(\begin{array}{l}
A_{n 2} \operatorname{BesselI}_{0}\left(m_{\eta} r\right)- \\
B_{n 2} \operatorname{BesselK}_{0}\left(m_{\eta} r\right)
\end{array}\right) \cos \left(m_{\eta} z\right)
\end{array}\right)
$$

$$
B_{r 3}=\sum_{\eta=1,2, \ldots}^{\infty} m_{\eta}\left(\begin{array}{l}
A_{\eta 3} \operatorname{BesselI}_{1}\left(m_{\eta^{r}}\right)+ \\
B_{\eta 3} \operatorname{BesselK}_{1}\left(m_{\eta^{r}}\right)
\end{array}\right) \sin \left(m_{\eta} z\right)
$$

$$
B_{z 3}=\sum_{\eta=1,2, \ldots}^{\infty} m_{\eta}\left(\begin{array}{l}
A_{\eta 3} \operatorname{BesselI}_{0}\left(m_{\eta} r\right)- \\
B_{\eta 3} \operatorname{BesselK}_{0}\left(m_{\eta} r\right)
\end{array}\right) \cos \left(m_{\eta} z\right)
$$

The last step is to find all coefficients in Equations (24) until (29). Solving these equations with all the boundary conditions in Equation (19) while considering Equations (1), (2) and (22) yields equations as functions of $r$ and $z$ only. Those equations represent the field distribution in the whole machine.

Induced Voltage: The flux linkage in the winding is computed by adding a coefficient, known as the Carter coefficient, to represent the existence of the stator teeth $[5,7]$. This coefficient is given by

$$
K_{C}=\frac{\tau_{s p}}{\tau_{s p}-\gamma g^{\prime}}
$$

where $\tau_{s p}$ is the stator slot pitch, and $g^{\prime}=g+h_{m} / \mu_{r}$. The slotting factor $\gamma$ is given by

$$
\gamma=\frac{4}{\pi}\left[\frac{b_{0}}{2 g^{\prime}} \tan ^{-1}\left(\frac{b_{0}}{2 g^{\prime}}\right)-\ln \sqrt{1+\left(\frac{b_{0}}{2 g^{\prime}}\right)^{2}}\right]
$$


where $b_{0}$ is the width of the stator slot openings.

Instead of the air gap distance g, an effective airgap $g_{\mathrm{e}}$ is used. This parameter is given by,

$$
g_{e}=g+\left(K_{C}-1\right) g^{\prime}
$$

Therefore, the stator bore radius should also be recalculated with the effect of slotting incorporated. The equivalent stator bore radius $R_{\mathrm{se}}$ is given by

$$
R_{i e}=R_{m}+g_{e}
$$

where $R_{m}$ is the outer radius of the magnets.

The flux linkage of a stator winding can be obtained by integrating the vector potential in the region 1 over the winding pitch. The total flux linkage of a phase winding is the sum of the flux linkages in all the windings which are connected in series.

$$
\Psi_{w}=\frac{N_{w}}{\tau_{w} \cdot\left(R_{S}-R_{i}\right)} \int_{z-\frac{\tau_{w}}{2}}^{z+\frac{\tau_{w}}{2}} \int_{R_{i}}^{R_{S}} 2 \cdot \pi \cdot r \cdot A_{I \theta}(r, z) d r d z
$$

The induced EMF in each phase winding is given by

$$
e_{w}=-\frac{d \Psi_{w}(z)}{d t}=-\frac{d \Psi_{w}(z)}{d z} v(t)
$$

where $v(t)$ is the velocity of the translator, in general, as a function of time.

\section{RESULTS}

The flux distribution in the air gap is calculated using Equations (24) and (25). The radius of location is setup to $r=R_{m}+g / 2$. The radial flux density $B_{r}$ and the tangential flux density $B_{z}$ are plotted in Fig. 3 together with the flux density resulted from the finite element analysis simulation.

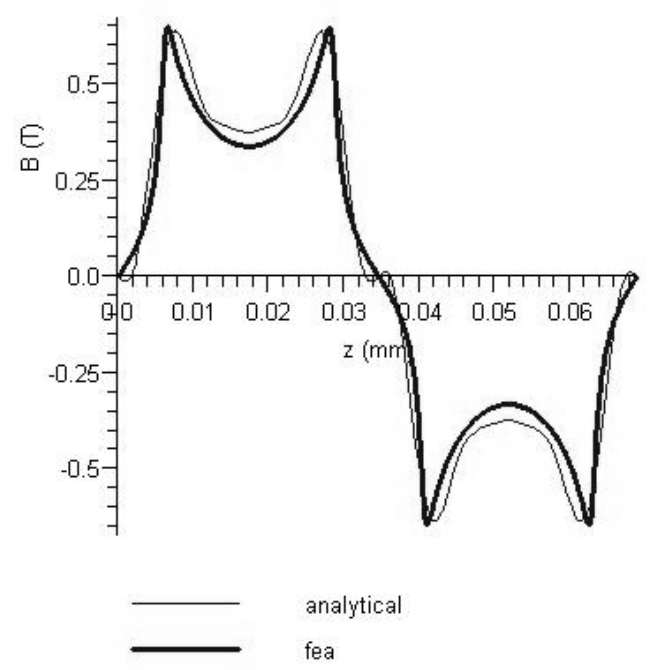

a. $B_{r}$

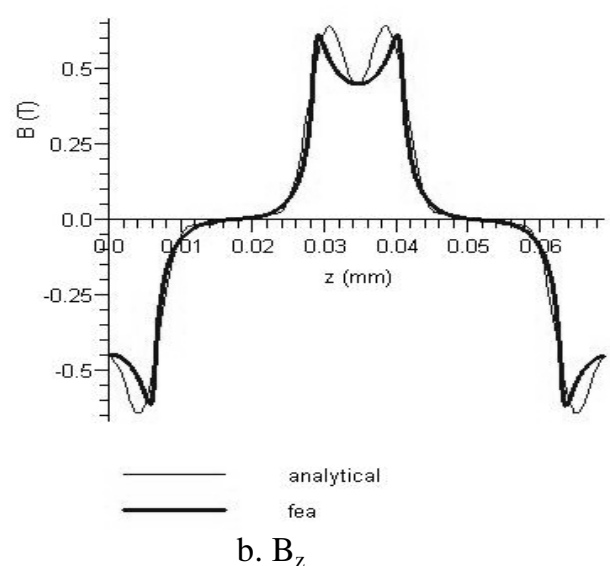

Fig. 3: Air gap flux density $B_{r}$ and $B_{z}$

The open circuit induced voltage $e_{w}$ calculated using Equation (35) and using the finite element analysis simulation are shown the Fig. 4.
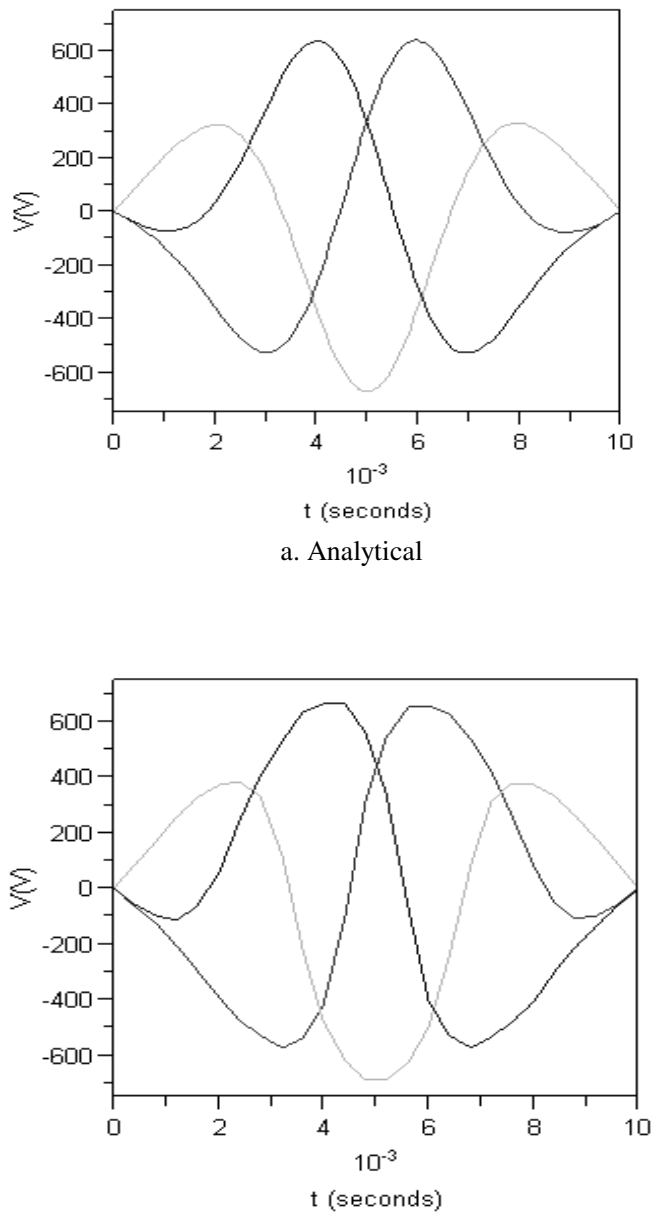

b. Finite Element Analysis

Fig. 4: Three Phase Induced Voltage 


\section{DISCUSSION}

Fig. 3 shows that the analytical result confirms to the simulation except that there is a little difference at some points. In the Fig. 4., the analytical results seem to agree well with the finite element analysis simulation. Consequently, other electrical parameters of the machine can be derived deductively from these parameters.

\section{CONCLUSIONS}

The analytical calculation of the open circuit magnetic field of a cylindrical permanent magnet linear generator is presented in this paper. The governing equation to the field problem is derived from the Laplace and Poisson equations. A special function known as Struve function is proposed to be used in solving the field equations. The solution is obtained without considering the geometrical condition of the permanent magnet as the field source.

The flux density in the air gap and open circuit induced voltage calculated using the analytical method are then compared to the ones produced by the finite element simulation. It can be seen that the results from the two methods match very well and therefore vindicate the validity of the analytical approach. It has been shown that the simple analytical method presented in this paper can be used as a tool for field estimation and design optimization in machine design.

\section{ACKNOWLEDGEMENT}

The authors gratefully appreciate and thank the Ministry Of Science, Technology and Environment, Malaysia, for the funding of this research project under IRPA Grant No.33-02-03-3013.

\section{REFERENCES}

1. H. Arof, Wijono and K. M. Nor, 2003, Linear Generator: Design and Simulation, in Proc. National Power Engineering Conference (PECon 2003), Bangi, Malaysia., pp. 306-311,

2. Ion Boldea, S.A. Nasar, Zhenxing Fu, 1988, Fields, Forces, and Performance Equations of Air-Core Linear Self-Synchronous Motor with Rectangular Current Control, IEEE Transactions On Magnetics, Vol. 24, pp. 2194-2203

3. Jiabin Wang, and David Howe, 2004, Design Optimization of Radially Magnetized, Iron-Cored, Tubular Permanent-Magnet Machines and Drive Systems, IEEE Transactions On Magnetics, Vol. 40, No. 5, pp. 3262-3277.

4. Jiabin Wang, David Howe, and Geraint W. Jewell, 2004, Analysis and Design Optimization of an Improved Axially Magnetized Tubular PermanentMagnet Machine, IEEE Transactions On Energy Conversion, Vol. 19, No. 2, pp. 289-295
5. Jiabin Wang, and David Howe, 2005, Tubular Modular Permanent-Magnet Machines Equipped With Quasi-Halbach Magnetized Magnets-Part I: Magnetic Field Distribution, EMF, and Thrust Force, IEEE Transactions on Magnetics, Vol. 41, No. 9, pp. 2470-2478.

6. Jiabin Wang, David Howe, and Geraint W. Jewell, 2003, Fringing in Tubular Permanent-Magnet Machines: Part I. Magnetic Field Distribution, Flux Linkage, and Thrust Force, IEEE Transactions on Magnetics, Vol. 39, No. 6, pp. 3507-3516.

7. Jiabin Wang, Geraint W. Jewell, and David Howe, 1999, A General Framework for the Analysis and Design of Tubular Linear Permanent Magnet Machines, IEEE Transactions on Magnetics, Vol. 35, No. 3, pp. 1986-2000.

8. Jiabin Wang; Howe, D., 2005 A Linear Permanent Magnet Generator for a Free-Piston Energy Converter, IEEE International Conference on Electric Machines and Drives, May 15, pp. 1521 1528

9. K. Ng, Z Q. Zhu and D Howe,1996, Open-Circuit Field Distribution in a Brushless Motor with Diametrically Magnetized PM Motor, Accounting for Eddy Current Effects, IEEE Transactions on Magnetics, Vol 32, No 5, pp. 5070-5072

10. Khalid Mohamed Nor, Hamzah Arof, and Wijono, 2004, Design of a $5 \mathrm{~kW}$ Tubular Permanent Magnet Linear Generator, in Proc. 39th International Universities Power Engineering Conference (UPEC 2004), University of the West of England (UWE) Bristol, UK., pp. 528-532

11. Khalid Mohamed Nor, Hamzah Arof, and Wijono, Design of a Three Phase Tubular Permanent Magnet Linear Generator, 2005, in Proc. The 5th Iasted International Conference On Power And Energy Systems, EuroPes 2005, Benalmádena, Spain

12. Z. J. Liu. C. Bi, H. C. Tan and T-S. Low, 1995, A Combined Numerical and Analytical Approach for Magnetic Field Analysis of Permanent Magnet Machines, IEEE Transactions on Magnetics. Vol. 31, No. 3., pp. 1372-1375

13. Z. P. Xia, Z. Q. Zhu, and D. Howe, 2004, Analytical Magnetic Field Analysis of Halbach Magnetized Permanent-Magnet Machines, IEEE Transactions on Magnetics, Vol. 40, No. 4, pp.1864-1872.

14. Z. Q. Zhu, D. Howe. and Z. P. Xia,, 1994, Prediction of Open-circuit Air gap Field Distribution Magnet Rotor Topology in Brushless Machines Having an Inset Permanent, IEEE Transactions on Magnetics, Vol. 30, No. I, pp.98107.

15. Z. Q. Zhu, David Howe, Ekkehard Bolte, and Bemd Ackermann, 1993, Instantaneous Magnetic Field Distribution in Brushless Permanent Magnet dc Motors, Part I: Open-circuit Field, IEEE Transactions on Magnetics, Vol. 29, No. 1, pp. 124-135.

16. Z. Q. Zhu, David Howe, Ekkehard Bolte, and Bemd Ackermann, 1993, Instantaneous Magnetic Field Distribution in Brushless Permanent Magnet dc Motors, Part III: Effect of Armature Slotting, IEEE Transactions on Magnetics, Vol. 29, No. 1, pp. 143-151. 